\title{
Trabajo en equipo y Flip Teaching para mejorar el aprendizaje activo del alumnado
}

\section{Teamwork and Flip Teaching to improve students’ active learning}

\author{
María Luisa Sein-Echaluce ${ }^{1}$, Ángel Fidalgo-Blanco ${ }^{2}$, Francisco J. García-Peñalvo ${ }^{3}$ \\ mlsein@unizar.es, angel.fidalgo@upm.es,fgarcia@usal.es
}

\author{
${ }^{1}$ Departamento de Matemática Aplicada \\ Universidad de Zaragoza \\ Madrid, España
}

\author{
${ }^{2}$ Departamento Ingeniería Geológica y \\ Minería \\ Universidad Politécnica de Madrid \\ Zaragoza, España
}

\author{
${ }^{3}$ Departamento de Informática y Automática \\ Universidad de Salamanca \\ Salamanca, España
}

\begin{abstract}
Resumen- Una de las formas de mejorar el aprendizaje en el alumnado es que este participe de forma activa en el proceso de formación. La metodología denominada Flip Teaching lleva las actividades del aula donde tiene una actitud pasiva (generalmente en las lecciones magistrales) a casa, donde también tiene una actitud pasiva visionando el vídeo del profesorado. Sin embargo, bajo el modelo Flip Teaching el alumnado realiza actividades activas y cooperativas. En este trabajo se modifica un modelo avanzado de Flip Teaching que permite al alumnado estar activo, también, en las actividades que se realizan fuera del aula. La modificación del modelo consiste en utilizar vídeos generados por el alumnado (en lugar de los vídeos del profesorado) a partir de su experiencia como estudiantes que ya han cursado la asignatura.
\end{abstract}

Palabras clave: Flip Teaching; Aprendizaje Activo; Aprendizaje Cooperativo, Aprendizaje por Pares, Inteligencia colectiva.

Abstract- One of the ways to improve students' learning is to promote that the student actively participates in the training process. The methodology called Flip Teaching takes the activities of the classroom, where students have a passive attitude (usually in the master lessons) at home, where they also have a passive attitude by watching the video of the teaching staff. However, under the Flip Teaching model the students perform active and cooperative activities. In this work, an advanced model of Flip Teaching is proposed, which allows the students to be active, also, in the activities that take place outside the classroom. The modification of the model consists in using videos generated by the students (instead of the videos of the teachers) based on their experience of students who have already taken the subject.

Keywords: Flip Teaching; Active Learning; Cooperative Learning: Peer Learning; Colective Intelligence.

\section{INTRODUCCIÓN}

El modelo Flip Teaching (FT) comenzó con el intercambio del lugar donde habitualmente se realizan dos de las principales actividades educativas: "la lección y los deberes" (Ramírez-Montoya \& Ramírez-Hernández, 2016). En este modelo la lección se realiza en casa y los deberes en clase. Lage, Platt y Treglia (2000) denominaron a este modelo Inverted Classroom y en las mismas fechas un modelo idéntico fue denominado por Baker (2000) como Classroom
Flip. Más tarde fueron Bergmann y Sams (2012) quienes lo denominaron Flipped Clasroom. A partir de esta fecha se ha ido consolidando el nombre de FT, aunque convive con los nombres iniciales.

Independientemente del nombre dado, el modelo FT se basa en un planteamiento básico de la gestión del conocimiento (Fidalgo-Blanco, Sein-Echaluce Lacleta, García-Peñalvo, \& Pinilla-Martínez, 2015; Fidalgo-Blanco, Sein-Echaluce, \& García-Peñalvo, 2014, 2015): la coincidencia física o temporal de personas genera conocimiento si hay interacción y participación activa en lugar de pasiva (Newton, Cameron, \& Ruiz Carillo De Albornoz, 2015).

Por otro lado, siguiendo este planteamiento, si el alumnado está pasivo durante una lección, el lugar donde se lleve a cabo es indiferente. Así pues, la "lección en casa” de FT suele estar formada por un vídeo grabado por el profesorado. El vídeo se suele complementar con foros para dudas, cuestionarios para comprobar que el alumnado ha "aprendido la lección” y material complementario. Así mismo, los vídeos deben cumplir un conjunto de requisitos para aumentar su eficacia, como la duración menor de 10 minutos (Medina, 2008).

Los “deberes en el aula” de FT suelen basarse en metodologías activas y cooperativas, como el aprendizaje basado en problemas, aprendizaje basado en casos o el trabajo en equipo.

A grandes rasgos, lo que hace el modelo FT es trasladar la inactividad del alumnado en el aula (que habitualmente se limita a escuchar la lección magistral) a su casa, ya que escuchar y ver el vídeo del profesor es una actividad igual o más inactiva.

Sin embargo, uno de los problemas del modelo FT es la posible desconexión e independencia de la "lección en casa" con los “deberes en el aula” (referencia). La responsabilidad de aprender la lección se atribuye al alumnado y en el aula es el profesorado el que se esfuerza en aplicar metodologías activas. Por tanto, si existe una desconexión (por ejemplo, si el alumnado no ha aprendido la lección) el modelo fracasa totalmente. 
En los últimos años han surgido modelos que tratan de conectar la actividad en casa con la actividad en clase y se suele añadir al modelo una actividad intermedia que mejora la actividad en casa. Es el caso del modelo MicroFlipTeaching (MFT) (Fidalgo-Blanco, Martinez-Nuñez, Borrás-Gene, \& Sánchez-Medina, 2017; Fidalgo-Blanco, Sein-Echaluce, \& García-Peñalvo, 2017; García-Peñalvo, Fidalgo-Blanco, SeinEchaluce Lacleta, \& Conde-González, 2016). En este modelo el alumnado realiza además un micro trabajo tras la visualización del vídeo y el profesorado utiliza los resultados de ese trabajo como recurso didáctico en el aula.

Esa actividad intermedia consigue que el alumnado, de forma individual o grupal, adquiera un conocimiento a través de la práctica; conocimiento que puede ser correcto, erróneo o incompleto. La variedad de conocimiento adquirido capacita al alumnado para realizar un aprendizaje entre iguales durante la sesión presencial en el aula, Dicho aprendizaje entre iguales es guiado, coordinado y gestionado por el profesorado. El modelo MFT se basa, por tanto, en el aprendizaje peer to peer durante la sesión de aula. En dichas sesiones se intercambia errores, enfoques y diversas formas de abordar una misma solución. Todos estos elementos son la base para que se produzca inteligencia colectiva.

El dialogo entre personas con distintos saberes, la interacción y la comunicación son los pilares del aprendizaje peer to peer, pero si se añade la dimensión tiempo, también son los pilares de la creación de inteligencia colectiva. El aprendizaje entre iguales fomenta una compresión más profunda de la interconexión de concepto lo que aporta un soporte para construir la inteligencia colectiva. Esta suele ser dinámica y permanente; es decir, no tiene un principio y un final acotado, ya que las personas aprenden y desaprenden de forma continua.

En la experiencia que aquí se presenta, se ha definido un grupo de control (CG) que utiliza la metodología MFT con vídeos generados por el profesorado en la actividad en casa. Así mismo, se ha definido un grupo experimental (EG) que utiliza vídeos en la actividad en casa que han sido generados por otro alumnado que ya ha adquirido el conocimiento en cursos anteriores.

El objetivo del trabajo es comprobar que se cumplen las siguientes condiciones respecto al aprendizaje basado en pares:

- En un mismo espacio de aprendizaje (el aula) convive alumnado que tanto de forma individual como grupal tiene distintos conocimientos e implicaciones sobre una materia concreta.

- Analizar la percepción del alumnado sobre los vídeos generados por el profesorado y los del alumnado.

- Que los resultados de aprendizaje del grupo experimental al menos es similar al grupo de control. De esta forma se estaría demostrando la eficacia del material generado por el alumnado que ya ha cursado la asignatura.

- Que los resultados de aprendizaje son, como mínimo, iguales entre el grupo CG y el EG. Así mismo se analizará si se dan las condiciones para que se produzca inteligencia colectiva.
En la siguiente sección se expone el modelo teórico del trabajo, se continuará con la descripción del contexto, se expondrán los resultados y se finalizará con las conclusiones.

\section{CONTEXTO}

La experiencia se ha realizado entre febrero y junio de 2017 en la asignatura “Informática y Programación”, del primer curso del Grado de Energía de la Universidad Politécnica de Madrid.

Han participado un total de 22 equipos de trabajo, con una media de 6 miembros por equipo. Para estudiar la incidencia del modelo se han dividido los equipos en dos grupos: el EG y el CG con 11 equipos cada uno. 132 alumnos han realizado la experiencia de un total de 180 alumnos de la asignatura. El alumnado que no ha participado en la experiencia no cumplía las mismas condiciones iniciales que el resto de alumnos y podían alterar los resultados. Se excluyeron los alumnos que ya habían realizado el trabajo en equipo en una anterior edición de la asignatura, grupos atípicos (por ejemplo, formado por 3 alumnos) y alumnos que comenzaron la asignatura con un retraso significativo respecto al resto de alumnos.

Los vídeos que utiliza el grupo experimental provienen de las lecciones aprendidas de alumnado de otra asignatura que realizaron de forma previa las mismas actividades. La asignatura se denomina "Fundamentos dela Programación”, de primer curso, primer semestre del grado de Biotecnología de la Universidad Politécnica de Madrid. Se debe observar que para la experiencia se utiliza conocimiento generado por alumnado de otro grado.

La primera sesión fue común para los equipos de los grupos EG y CG. Para esta primera fase se utilizaron tanto los vídeos del profesorado como del alumnado; es decir, todo el alumnado participante en la experiencia tenía acceso a los mismos vídeos.

A partir de la segunda sesión y hasta la quinta (y última) el grupo CG únicamente accedió a los vídeos del profesorado y el grupo EG a los del alumnado ¿̇además o en lugar de los del profesorado?

\section{DESCRIPCIÓN}

A través de la bibliografía se pueden clasificar los modelos FT en dos modalidades: los modelos que simplemente trasladan la pasividad del alumnado de dentro a fuera del aula y los modelos que incrementan la actividad tanto fuera como dentro del aula.

Otros modelos potencian la actividad del alumnado tanto fuera como dentro del aula. Uno de esos modelos el MFT citado con anterioridad el que se aplica en este trabajo.

El modelo se refleja en la Figura 1, consta de tres actividades:

Actividad en Casa: se trata de emular un entorno de formación presencial durante una sesión magistral. Se dispone de un vídeo explicativo (simula la explicación del profesorado) un foro para preguntas y respuestas (simula la interacción alumnado-profesorado) para resolver dudas y material complementario para realizar la actividad intermedia. 
Actividad intermedia: El alumnado organizado en equipos de trabajo realiza la actividad propuesta en la actividad anterior.

Actividad en clase: El alumnado comparte las distintas soluciones y la experiencia para llegar a ella. Se trabaja tanto con las soluciones erróneas como correctas. Durante esta actividad los equipos corrigen sus propias soluciones. Posteriormente el profesorado imparte mini-lecciones magistrales para reforzar la acción o introducir la próxima sesión.

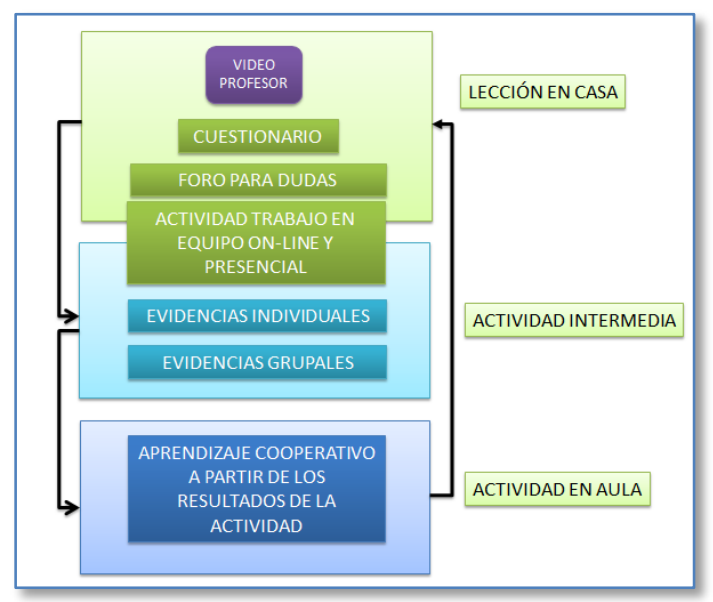

Figura 1. Modelo activo MFT

El modelo MFT planta que la fuente emisora de conocimiento para la fase inicial (actividad en casa) lo hace el propio profesorado de la asignatura. En este trabajo se modifica el modelo cambiando la fuente emisora de dicho conocimiento. EN el grupo experimental la fuente emisora es alumnado que ya ha cursado dicha materia y en el de control el profesorado. La Figura 2 refleja esta modificación al modelo. Dicho modelo se denomina Active Peer-Based Flip Teaching (APFT).

El método seguido para ambos grupos consistió:

Actividad en casa: vídeos conceptuales (grupo de control creado por el profesorado y el grupo experimental por el alumnado) y explicaciones de la actividad intermedia a realizar (parte de un trabajo en equipo).

Actividad intermedia: actividad en realizar mediante trabajo en equipo. El alumnado, fuera del aula, trabaja en equipo para resolver la actividad propuesta. Para los debates utilizan un foro y las conclusiones del trabajo las pone cada equipo en Wiki-Moodle. Tanto el foro como la wiki son privadas para cada equipo.

Actividad en aula: Los equipos exponen sus conclusiones, algunos equipos tienen el resultado incorrecto, otros correcto y otros equipos hace falta alguna mejora. A partir de las exposiciones y debate cada equipo mejora su solución aportada. Esta técnica se basa en aprendizaje entre iguales e inteligencia colectiva, ya que se intercambian los distintos saberes de las personas para buscar una solución común.

Así pues, para el grupo experimental, en una actividad de aprendizaje (actividad intermedia) se propone utilizar el conocimiento de alumnos de otras asignaturas, titulaciones, universidades e incluso de otros cursos académicos. De esta forma se construye inteligencia colectiva durante la actividad en casa del modelo genérico FT.

A continuación se exponen los resultados de la experiencia.

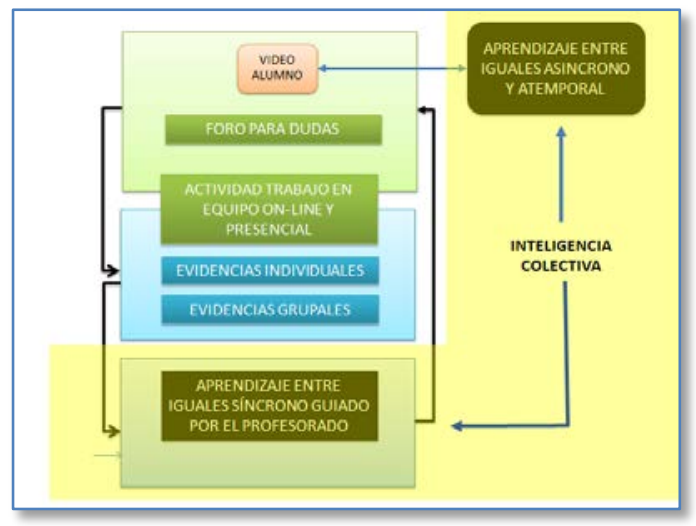

Figura 1. Modelo APFT

\section{RESUltados}

\section{Resultados de seguimiento}

El día anterior a cada sesión de aula (correspondiente a cada fase del trabajo en equipo) se realizó una evaluación del grado de consecución de los resultados esperados en cada fase del trabajo. Se detectaron los miembros de cada equipo con una baja carga de trabajo ("posibles jetas”) y los resultados esperados obtenidos.

Los resultados se expresan de forma gráfica, tal y como muestra la Figura 3. En la primera columna se indica el nombre del equipo

\begin{tabular}{|l|c|c|c|c|c|}
\hline & \multicolumn{1}{|c|}{ INDIVIDUAL } & FASE I & FASE II & FASE III & FASE IV \\
\hline 1 GIE2 & IY & CG & CG & CG & CR \\
\hline 10 GIE2 & IO & CG & CG & CY & CR \\
\hline 11 GIE2 & IG & CG & CG & CG & CG \\
\hline 12 GIE2 & IO & CG & CG & CG & CR \\
\hline 2 GIE2 & IG & CG & CG & CG & CG \\
\hline 3 GIE2 & IY & CG & CY & CG & CR \\
\hline 4 GIE2 & IG & CG & CG & CG & CG \\
\hline 5 GIE2 & IY & CG & CG & CG & CG \\
\hline 6 6IE2 & IO & CG & CG & CG & CG \\
\hline 7 7GIE2 & IY & CG & CG & CG & CG \\
\hline 9GIE2 & IO & CR & CR & CR & CR \\
\hline \hline
\end{tabular}

Figura 3. Ejemplo de tabla de seguimiento correspondiente a la sesión 4 (fase de ejecución)

La segunda columna indica la distribución de la carga de trabajo de los distintos miembros del equipo y el resto de columnas son las distintas fases grupales que se deben realizar. Los colores significan:

- Verde (IG). Hay una distribución de trabajo homogénea entre los miembros del equipo.

- Amarillo (IY). Hay una distribución homogénea pero hay personas situadas en el rango superior y otras en el rango inferior, dentro de la homogeneidad. 
- Naranja (IO). Hay personas que trabajan poco y otras más.

- $\quad$ Roja (IR). Hay jetas, hay personas que no están trabajando en el equipo.

Respecto a las fases (columnas 3, 4, 5 y 6) los colores indican:

- $\quad$ Verde (CG). el grado de desarrollo es correcto.

- Amarillo(CY). El grado de desarrollo es correcto, pero hay que hacer alguna modificación no importante.

- Naranja (CO). El desarrollo se ha iniciado pero hay alguna falta grave.

- $\quad$ Rojo (CR). O bien no se ha iniciado o está mal.

Los resultados para el reparto homogéneo de la carga se expresan en la Tabla 1.

\section{Tabla 1}

Reparto de la carga de trabajo individual a lo largo del desarrollo del trabajo en equipo

\begin{tabular}{ccc}
\hline Sesión & EG & CG \\
\hline Verde & 16 & 14 \\
Amarillo & 13 & 10 \\
Naranja & 7 & 8 \\
Rojo & 8 & 12 \\
\hline
\end{tabular}

Respecto a las fases, el grado de consecución global de las mismas se expresa en la Tabla 2.
Tabla 2

Progreso de consecución de las fases a lo largo del desarrollo del curso

$\begin{array}{ccc}\text { Sesión } & \text { EG } & \text { CG } \\ \text { Verde } & 68 & 52 \\ \text { Amarillo } & 10 & 24 \\ \text { Naranja } & 4 & 8 \\ \text { Rojo } & 22 & 20\end{array}$

\section{Resultados de aprendizaje}

Se basan en la nota final obtenida por los distintos equipos de trabajo al finalizar. En la Tabla 3 se incluye el número de equipos (de EG y de CG) que han obtenido una calificación final dentro del rango correspondiente.

Tabla 3

Calificaciones finales de los equipos (sobre 6 puntos)

\begin{tabular}{lcc}
\hline & EG & CG \\
Rango 1 y 2,9 & 2 & 2 \\
Rango 3 y 4,9 & 5 & 6 \\
Rango 5 y 6 & 4 & 3 \\
Nota media - Desviación & $4,5-1,09$ & $4,2-1,4$ \\
\hline
\end{tabular}

\section{Resultados de percepción respecto a los vídeos}

Se realizó una encuesta donde participaron 88 personas (de 132) sobre la percepción sobre las preferencias del vídeo a utilizar obtenidas de la primera sesión (se utilizaban vídeos del profesorado y del alumnado). Los resultados se pueden encontrar en la Figura 4.

\begin{tabular}{|c|c|c|c|c|c|c|c|}
\hline & & & & \multicolumn{3}{|c|}{ Ranking de la media } & ॥ \\
\hline & & 1 & 2 & 3 & 4 & 5 & \\
\hline Prefiero los videos del profesorado. & & & \multicolumn{4}{|c|}{ - } & 3.2 \\
\hline Prefiero los videos del alumnado. & & & \multicolumn{4}{|c|}{ - } & 3.1 \\
\hline Prefiero que se dispongan videos del profesorado + alumnado & & & & \multicolumn{3}{|r|}{ 1 } & 4.5 \\
\hline Respuestas & 1 & 2 & 3 & \multicolumn{2}{|l|}{4} & 5 & Total \\
\hline Prefiero los videos del profesorado. & $5(6 \%)$ & $14(16 \%)$ & $35(40 \%)$ & \multicolumn{2}{|c|}{$23(26 \%)$} & $11(13 \%)$ & 88 \\
\hline Prefiero los videos del alumnado. & $6(7 \%)$ & $17(19 \%)$ & $36(41 \%)$ & \multicolumn{2}{|c|}{$23(26 \%)$} & $6(7 \%)$ & 88 \\
\hline Prefiero que se dispongan videos del profesorado + alumnado & 0 & 0 & $11(13 \%)$ & \multicolumn{2}{|c|}{$18(20 \%)$} & $59(67 \%)$ & 88 \\
\hline
\end{tabular}

Figura 4. Preferencias sobre el tipo de vídeo

\section{CONCLUSIONES}

Respecto a las condiciones para que se produzca el aprendizaje peer to peer durante la actividad de aula se puede observar que tanto a nivel individual (Tabla 1) como grupal (tabla 2) se demuestra que las personas que participan en una misma sesión presencial tienen conocimientos e implicaciones distintas respecto a una materia determinada. Por ejemplo, en la tabla 1 se puede observar que los miembros del equipo han tenido distinta implicación respecto al trabajo ene quipo realizado en la actividad intermedia, por tienen distinto 
conocimiento individual. Lo mismo ocurre con los equipos de trabajo, no todos los equipos han realizado las distintas fases del trabajo de forma correcta. Así pues, cada alumno y cada equipo tiene distintos saberes y niveles de experiencia. Esta condición es la base para que se produzca aprendizaje a través de la interacción, ya que si todos tuviesen el mismo nivel de conocimiento no se produciría ningún aprendizaje.

Respecto a la eficacia de la utilización de los vídeos generados por el alumnado, se demuestra que las puntuaciones obtenidas en el trabajo final son muy similares, aunque son algo superiores las de grupo experimental no hay diferencias significativas. Este hecho demuestra la viabilidad de los vídeos generados por el alumnado como recurso didáctico a utilizar en la actividad en casa de Flip Teaching y, por tanto, valida el modelo APFT propuesto en este trabajo de investigación.

En la primera sesión se suministró a los dos grupos tanto el conocimiento generado por el profesorado como el generado por el alumnado. En la Figura 4 el alumnado no muestra una diferencia significativa por el conocimiento del profesorado o por el alumnado, este hecho complementa a los resultados de aprendizaje, indicando la validez del vídeo generado por el alumnado. Sin embargo, si hay una diferencia muy significativa al apostar porque prefieren los dos tipos de vídeos (los generados por el profesorado como los generados por el alumnado). Esta preferencia es propia de la inteligencia colectiva, ya que por un lado se dice que es necesario el conocimiento del alumnado, pero también del profesorado.

Así pues se demuestra que:

- El aprendizaje del alumnado se puede encapsular y utilizar bajo el APFT en cursos posteriores, aun partiendo de asignaturas y titulaciones distintas.

- $\quad$ Se puede plantear un proceso de formación, bajo las condiciones del modelo APFT, como un proceso de construcción de inteligencia colectiva a partir de los distintos saberes del alumnado, del profesorado $\mathrm{y}$ exalumnado.

En este trabajo se ha utilizado conocimiento encapsulado del semestre anterior de una asignatura y titulación distinta a la asignatura donde se realizó la experiencia. Pero cabe plantearse si el conocimiento encapsulado de los alumnos es más útil si lo genera el de una misma asignatura que el que se genera en otras asignaturas.

Por otra parte, la gestión de los recursos generados en el proceso de inteligencia colectiva se incrementa con el tiempo, y ya en cada utilización del modelo se incrementa de forma lineal el número de personas que participan, por tanto ¿crecerá el conocimiento también de forma lineal?, ¿se complicará la gestión del incremento de recursos de conocimiento?

Todos estos planteamientos guiarán nuevas líneas de trabajo futuro.

\section{AgRADECIMIENTOS}

Los autores quieren agradecer al proyecto de Innovación Educativa con código IE1617.0601 de la UnivERSIDAD POLITÉCNICA DE MADRID. Al Gobierno de Aragón, el Fondo Social Europeo, y el Ministerio de Educación de la Región de Castilla y León por su apoyo, así como a los grupos de investigación (LITI, http://www.liti.es; http://gidtic.com y GRIAL, http://grial.usale.es).

Este trabajo está parcialmente financiado por el Ministerio de Economía y Competitividad del Gobierno de España a través del proyecto DEFINES (Ref. TIN2016-80172-R).

\section{REFERENCIAS}

Baker, J. W. (2000). The 'Classroom Flip': Using Web Course Management Tools to Become the Guide by the Side. In J. A. Chambers (Ed.), Selected Papers from the 11th International Conference on College Teaching and Learning (pp. 9-17). Jacksonville, FL: Florida: Community College at Jacksonville.

Bergmann, J., \& Sams, A. (2012). Flip Your Classroom: Reach Every Student in Every Class Every Day. New York, NY: Buck Institute for International Society for Technology in Education.

Fidalgo-Blanco, Á., Martinez-Nuñez, M., Borrás-Gene, O., \& Sánchez-Medina, J. J. (2017). Micro flip teaching An innovative model to promote the active involvement of students. Computers in Human Behavior, 72, 713-723. doi:https://doi.org/10.1016/j.chb.2016.07.060

Fidalgo-Blanco, Á., Sein-Echaluce Lacleta, M. L., GarcíaPeñalvo, F. J., \& Pinilla-Martínez, J. (2015). BRACO: Buscador de Recursos Académicos Colaborativos. In Á. Fidalgo Blanco, M. L. SeinEchaluce Lacleta, \& F. J. García-Peñalvo (Eds.), La Sociedad del Aprendizaje. Actas del III Congreso Internacional sobre Aprendizaje, Innovación y Competitividad. CINAIC 2015 (14-16 de Octubre de 2015, Madrid, España) (pp. 469-474). Madrid, Spain: Fundación General de la Universidad Politécnica de Madrid.

Fidalgo-Blanco, Á., Sein-Echaluce, M. L., \& García-Peñalvo, F. J. (2014). Knowledge Spirals in Higher Education Teaching Innovation. International Journal of Knowledge Management, 10(4), 16-37. doi:10.4018/ijkm.2014100102

Fidalgo-Blanco, Á., Sein-Echaluce, M. L., \& García-Peñalvo, F. J. (2015). Epistemological and ontological spirals: From individual experience in educational innovation to the organisational knowledge in the university sector. Program: Electronic library and information systems, 49(3), 266-288. doi:10.1108/PROG-062014-0033

Fidalgo-Blanco, Á., Sein-Echaluce, M. L., \& García-Peñalvo, F. J. (2017). Ontological Flip Teaching: a Flip Teaching model based on knowledge management. Universal Access in the Information Society, In Press. doi:10.1007/s10209-017-0556-6

García-Peñalvo, F. J., Fidalgo-Blanco, Á., Sein-Echaluce Lacleta, M., \& Conde-González, M. Á. (2016). Cooperative Micro Flip Teaching. In P. Zaphiris \& I. Ioannou (Eds.), Learning and Collaboration Technologies. Third International Conference, LCT 2016, Held as Part of HCI International 2016, Toronto, ON, Canada, July 17-22, 2016, Proceedings 
(pp. 14-24). Switzerland: Springer International Publishing.

Lage, M. J., Platt, G. J., \& Treglia, M. (2000). Inverting the Classroom: A Gateway to Creating an Inclusive Learning Environment. The Journal of Economic Education, 31(1), 30-43.

Medina, J. (2008). Brain Rules: 12 Principles for surviving and thriving at Work, Home, and School. Seattle, WA: Pear Press.

Newton, C., Cameron, R., \& Ruiz Carillo De Albornoz, A. (2015). Flipped teaching: finding room for interdisciplinary content and peer learning Living and
Learning: Research for a Better Built Environment: 49th International Conference of the Architectural Science Association (pp. 967-976). Melbourne, Australia: The Architectural Science Association and The University of Melbourne.

Ramírez-Montoya, M. S., \& Ramírez-Hernández, D. C. (2016). Inverted Learning Environments with Technology, Innovation and Flexibility: Student experiences and meanings. Journal of Information Technology Research, 9(1), 18-33. doi:10.4018/JITR.2016010102 